\title{
Multiset filters
}

\author{
Amr Zakaria ${ }^{1,2^{*}}$ (D), Sunil Jacob John ${ }^{3}$ and K. P. Girish ${ }^{4}$
}

\section{${ }^{*}$ Correspondence:}

amr.zakaria@edu.asu.edu.eg 'Doctoral School of Mathematical and Computational Sciences, University of Debrecen, Pf. 400, Debrecen, $\mathrm{H}-4002$, Hungary ${ }^{2}$ Department of Mathematics, Faculty of Education, Ain Shams University, Cairo 11341, Egypt Full list of author information is available at the end of the article

\begin{abstract}
A multiset is a collection of objects in which repetition of elements is essential. This paper is an attempt to generalize the notion of filters in the multiset context. In addition, many deviations between multiset filters and ordinary filters have been presented. The relation between multiset filter and multiset ideal has been mentioned. Many properties of multiset filters, multiset ultrafilters, and convergence of multiset filters have been introduced. Also, the notions of basis and subbasis have been mentioned in the multiset context. Finally, several examples have been studied.

Keywords: Multiset, Multiset filter, Multiset ultrafilter, Multiset topology

Mathematics Subject Classification (2010): 54A05; 03E70
\end{abstract}

\section{Introduction}

In classical set theory, a set is a well-defined collection of distinct objects. If repeated occurrences of any object is allowed in a set, then a mathematical structure is known as multiset (mset [1] or bag [2], for short). Thus, an mset differs from a set in the sense that each element has a multiplicity and a natural number not necessarily one that indicates how many times it is a member of the mset. One of the most natural and simplest examples is the mset of prime factors of a positive integer $n$. The number 400 has the factorization $400=2^{4} 5^{2}$ which gives the mset $\{2,2,2,2,5,5\}$. Also, the cubic equation $x^{3}-5 x^{2}+3 x+9=0$ has roots 3,3 , and -1 which give the mset $\{3,3,-1\}$.

Classical set theory is a basic concept to represent various situations in mathematical notation where repeated occurrences of elements are not allowed. But in various circumstances, repetition of elements becomes mandatory to the system. For example, in a graph with loops, there are many hydrogen atoms, many water molecules, many strands of identical DNA, etc. This leads to effectively three possible relations between any two physical objects: they are different, they are the same but separate, or they are coinciding and identical. For example, ammonia $\mathrm{NH}_{3}$ has with three hydrogen atoms, say $\mathrm{H}, \mathrm{H}$, and $H$, and one nitrogen atom, say $N$. Clearly, $H$ and $N$ are different. However $H, H$, and $H$ are the same but separate, while $H$ and $H$ are coinciding and identical. There are many other examples, for instance, carbon dioxide $\mathrm{CO}_{2}$, sulfuric acid $\mathrm{H}_{2} \mathrm{SO}_{4}$, and water $\mathrm{H}_{2} \mathrm{O}$.

This paper is an attempt to explore the theoretical aspects of msets by extending the notions of filters, ultrafilters, and convergence of filters to the mset context. The "Preliminaries and basic definitions" section has a collection of all basic definitions and notions for further study. In the "On multiset topologies" section, examples of new mset topologies are introduced. In the "Filters in multiset context" section, the notion of mset

(c) The Author(s). 2019 Open Access This article is distributed under the terms of the Creative Commons Attribution 4.0 International License (http://creativecommons.org/licenses/by/4.0/), which permits unrestricted use, distribution, and reproduction in any medium, provided you give appropriate credit to the original author(s) and the source, provide a link to the Creative Commons license, and indicate if changes were made. 
filters has been introduced. Further, many properties of this notion have been mentioned. In the "Basis and subbasis in multiset filters" section, basis and subbasis of mset filters are mentioned. In the "Multiset ultrafilter" section, the concept of mset ultrafilter has been presented and several examples and properties of this notion are introduced. In the "Convergence of multiset filters" section, convergence of mset filters and its properties are studied.

\section{Preliminaries and basic definitions}

In this section, a brief survey of the notion of msets as introduced by Yager [2], Blizard $[1,3]$, and Jena et al. [4] have been collected. Furthermore, the different types of collections of msets, the basic definitions, and notions of relations and functions in mset context are introduced by Girish and John [5-8]. Other important research about multiset theory and its applications can be found in [9-16].

Definition 1 A collection of elements containing duplicates is called an mset. Formally, if $X$ is a set of elements, an mset $M$ drawn from the set $X$ is represented by a function count $M$ or $C_{M}$ defined as $C_{M}: X \rightarrow \mathbb{N}$ where $\mathbb{N}$ represents the set of nonnegative integers.

Let $M$ be an mset from the set $X=\left\{x_{1}, x_{2}, \ldots, x_{n}\right\}$ with $x$ appearing $n$ times in $M$. It is denoted by $x \in \in^{n} M$. The mset $M$ drawn from the set $X$ is denoted by $M=$ $\left\{k_{1} / x_{1}, k_{2} / x_{2}, \ldots, k_{n} / x_{n}\right\}$ where $M$ is an mset with $x_{1}$ appearing $k_{1}$ times, $x_{2}$ appearing $k_{2}$ times, and so on. In Definition $10, C_{M}(x)$ is the number of occurrences of the element $x$ in the mset $M$. However, those elements which are not included in the mset $M$ have zero count. An mset $M$ is a set if $C_{M}(x)=0$ or $1 \forall x \in X$.

Definition $2 A$ domain $X$ is defined as a set of elements from which msets are constructed. The mset space $[X]^{m}$ is the set of all msets whose elements are in $X$ such that no element in the mset occurs more than $m$ times. The set $[X]^{\infty}$ is the set of all msets over a domain $X$ such that there is no limit on the number of occurrences of an element in an mset.

Let $M, N \in[X]^{m}$. Then, the following are defined:

(1) $\quad M$ is a submset of $N$ denoted by $(M \subseteq N)$ if $C_{M}(x) \leq C_{N}(x) \forall x \in X$.

(2) $M=N$ if $M \subseteq N$ and $N \subseteq M$.

(3) $\quad M$ is a proper submset of $N$ denoted by $(M \subset N)$ if $C_{M}(x) \leq C_{N}(x) \forall x \in X$ and there exists at least one element $x \in X$ such that $C_{M}(x)<C_{N}(x)$.

(4) $\quad P=M \cup N$ if $C_{P}(x)=\max \left\{C_{M}(x), C_{N}(x)\right\}$ for all $x \in X$.

(5) $\quad P=M \cap N$ if $C_{P}(x)=\min \left\{C_{M}(x), C_{N}(x)\right\}$ for all $x \in X$.

(6) Addition of $M$ and $N$ results is a new mset $P=M \oplus N$ such that $C_{P}(x)=\min \left\{C_{M}(x)+C_{N}(x), m\right\}$ for all $x \in X$.

(7) Subtraction of $M$ and $N$ results in a new mset $P=M \ominus N$ such that $C_{P}(x)=\max \left\{C_{M}(x)-C_{N}(x), 0\right\}$ for all $x \in X$, where $\oplus$ and $\ominus$ represent mset addition and mset subtraction, respectively.

(8) An mset $M$ is empty if $C_{M}(x)=0 \forall x \in X$.

(9) The support set of $M$ denoted by $M^{*}$ is a subset of $X$ and $M^{*}=\left\{x \in X: C_{M}(x)>0\right\}$; that is, $M^{*}$ is an ordinary set and it is also called root set. 
(10) The cardinality of an mset $M$ drawn from a set $X$ is $\operatorname{Card}(M)=\sum_{x \in X} C_{M}(x)$.

(11) $M$ and $N$ are said to be equivalent if and only if $\operatorname{Card}(M)=\operatorname{Card}(N)$.

Definition 3 Let $M \in[X]^{m}$ and $N \subseteq M$. Then, the complement $N^{c}$ of $N$ in $[X]^{m}$ is an element of $[X]^{m}$ such that $N^{c}=M \ominus N$.

Definition 4 A submset $N$ of $M$ is a whole submset of $M$ with each element in $N$ having full multiplicity as in $M$; that is, $C_{N}(x)=C_{M}(x)$ for every $x \in N^{*}$.

Definition 5 A submset $N$ of $M$ is a partial whole submset of $M$ with at least one element in $N$ having full multiplicity as in M. i.e., $C_{N}(x)=C_{M}(x)$ for some $x \in N^{*}$.

Definition 6 A submset $N$ of $M$ is a full submset of $M$ if each element in $M$ is an element in $N$ with the same or lesser non-zero multiplicity as in $M$, i.e., $M^{*}=N^{*}$ with $C_{N}(x) \leq$ $C_{M}(x)$ for every $x \in N^{*}$.

Definition 7 Let $M \in[X]^{m}$. The power whole mset of $M$ denoted by $P W(M)$ is defined as the set of all whole submsets of $M$.

Definition 8 Let $M \in[X]^{m}$. The power full msets of $M, P F(M)$, is defined as the set of all full submsets of $M$. The cardinality of PF $(M)$ is the product of the counts of the elements in $M$.

Definition 9 Let $M \in[X]^{m}$. The power mset $P(M)$ of $M$ is the set of all submsets of $M$. We have $N \in P(M)$ if and only if $N \subseteq M$. If $N=\phi$, then $N \in{ }^{1} P(M)$, and if $N \neq \phi$, then $N \in \epsilon^{k} P(M)$ such that $k=\prod_{z}\left(\begin{array}{l}\left|[M]_{z}\right| \\ \left|[N]_{z}\right|\end{array}\right)$, the product $\prod_{z}$ is taken over distinct elements of the mset $N$ and $\left|[M]_{z}\right|=m$ iff $z \in^{m} M,\left|[N]_{z}\right|=n$ iff $z \in^{n} N$, then $\left(\begin{array}{c}\left|[M]_{z}\right| \\ \left|[N]_{z}\right|\end{array}\right)=\left(\begin{array}{c}m \\ n\end{array}\right)=\frac{m !}{n !(m-n) !}$.

The power set of an mset is the support set of the power mset and is denoted by $P^{*}(M)$. The following theorem shows the cardinality of the power set of an mset.

Definition 10 Let $M_{1}$ and $M_{2}$ be two msets drawn from a set $X$, then the Cartesian product of $M_{1}$ and $M_{2}$ is defined as $M_{1} \times M_{2}=\left\{(m / x, n / y) / m n: x \in^{m} M_{1}, y \in \in^{n} M_{2}\right\}$.

Here, the entry $(m / x, n / y) / m n$ in $M_{1} \times M_{2}$ denotes $x$ is repeated $m$ times in $M_{1}, y$ is repeated $n$ times in $M_{2}$, and the pair $(x, y)$ is repeated mn times in $M_{1} \times M_{2}$.

Definition 11 A submset $R$ of $M_{1} \times M_{2}$ is said to be an mset relation on $M$ if every member $(m / x, n / y)$ of $R$ has a count, the product of $C_{1}(x, y)$ and $C_{2}(x, y) . m / x$ related to $n / y$ is denoted by $(m / x) R(n / y)$. The domain of the mset relation $R$ on $M$ is defined as follows:

$$
\begin{aligned}
& \operatorname{Dom}(R)=\left\{x \in^{r} M: \exists y \in^{s} M \text { such that }(r / x) R(s / y)\right\}, \text { where } \\
& C_{\operatorname{Dom}(R)}(x)=\operatorname{Sup}\left\{C_{1}(x, y): x \in^{r} M\right\} .
\end{aligned}
$$

Also, the range of the mset relation $R$ on $M$ is defined as follows: 


$$
\begin{aligned}
& \operatorname{Ran}(R)=\left\{y \in^{s} M: \exists x \in^{r} M \text { such that }(r / x) R(s / y)\right\}, \text { where } \\
& C_{\text {Ran (R) }}(y)=\operatorname{Sup}\left\{C_{2}(x, y): y \in^{s} M\right\} .
\end{aligned}
$$

Definition 12 An mset relation $f$ is called an mset function if for every element $m / x$ in Dom $f$, there is exactly one $n / y$ in Ran $f$ such that $(m / x, n / y)$ is in $f$ with the pair occurring as the product of $C_{1}(x, y)$ and $C_{2}(x, y)$.

Definition 13 An mset function $f$ is one-one (injective) if no two elements in Dom $f$ have the same image under $f$ with $C_{1}(x, y) \leq C_{2}(x, y)$ for all $(x, y)$ in $f$, i.e., if $m_{1} / x_{1}, m_{2} / x_{2}$ in Dom $f$ and $m_{1} / x_{1} \neq m_{2} / x_{2}$ implies that $f\left(m_{1} / x_{1}\right) \neq f\left(m_{2} / x_{2}\right)$. Thus, the one-one mset function is the mapping of the distinct elements of the domain to the distinct elements of the range.

Definition 14 An mset function $f$ is onto (surjective) if Ran $f$ is equal to co-dom $f$ and $C_{1}(x, y) \geq C_{2}(x, y)$ for all $(x, y)$ in $f$. It may be noted that images of distinct elements of the domain need not be distinct elements of the range.

Definition 15 Let $M$ be an mset drawn from a set $X$ and $\tau \subseteq P^{*}(M)$. Then, $\tau$ is called an mset topology if $\tau$ satisfies the following properties:

(1) $\phi$ and $M$ are in $\tau$.

(2) The union of the elements of any subcollection of $\tau$ is in $\tau$.

(3) The intersection of the elements of any finite subcollection of $\tau$ is in $\tau$.

An mset topological space is an ordered pair $(M, \tau)$ consisting of an mset $M$ and an mset topology $\tau \subseteq P^{*}(M)$ on $M$. Note that $\tau$ is an ordinary set whose elements are msets and the mset topology is abbreviated as an $M$-topology. Also, a submset $U$ of $M$ is an open mset of $M$ if $U$ belongs to the collection $\tau$. Moreover, a submset $N$ of $M$ is closed mset $M \ominus N$ is open mset.

Definition 16 Let $(M, \tau)$ be an M-topological space and $N$ be a submset of $M$. Then, the interior of $N$ is defined as the mset union of all open msets contained in $N$ and is denoted by $N^{o}$; that is, $N^{o}=\cup\{V \subseteq M: V$ is an open mset and $V \subseteq N\}$ and $C_{N^{o}}(x)=\max \left\{C_{V}(x)\right.$ : $V \subseteq N\}$.

Definition 17 Let $(M, \tau)$ be an $M$-topological space and $N$ be a submset of $M$. Then, the closure of $N$ is defined as the mset intersection of all closed msets containing $N$ and is denoted by $\bar{N}$; that is, $\bar{N}=\cap\{K \subseteq M: K$ is a closed mset and $N \subseteq K\}$ and $C_{\bar{N}}(x)=$ $\min \left\{C_{K}(x): N \subseteq K\right\}$.

Definition 18 An mset $M$ is called simple if all its elements are the same. For example, $\{k / x\}$. In addition, $k / x$ is called simple multipoint (for short mpoint).

Definition 19 Let $(M, \tau)$ be a M-topological space, $x \in^{k} M$, and $N \subseteq M$. Then, $N$ is said to be a neighborhood of $k / x$ if there is an open mset $V$ in $\tau$ such that $x \epsilon^{k} V$ and $C_{V}(y) \leq C_{N}(y)$ for all $y \neq x$ that is, $\mathcal{N}_{k / x}=\left\{N \subseteq M: \exists V \in \tau\right.$ such that $x \in^{k}$ $V$ and $C_{V}(y) \leq C_{N}(y)$ for all $\left.y \neq x\right\}$ is the collection of all $\tau$-neighborhood of $k / x$. 


\section{On multiset topologies}

Theorem 1 Let $f: M_{1} \longrightarrow M_{2}$ be an mset function, $V \subseteq M_{2}$ and $N \subseteq M_{1}$. Then:

(1) $f^{-1}\left(M_{2} \ominus V\right)=f^{-1}\left(M_{2}\right) \ominus f^{-1}(V)$.

(2) $N \subseteq f^{-1}(f(N))$, equality holds if $f$ is one-one.

(3) $f\left(f^{-1}(V)\right) \subseteq V$, equality holds if $f$ is onto.

Proof (1) Let $x \in^{k} f^{-1}\left(M_{2} \ominus V\right)$. Hence $f(k / x) \in\left(M_{2} \ominus V\right)$. So $f(k / x) \notin V$; that is, $k / x \in f^{-1}\left(M_{2}\right)$ and $k / x \notin f^{-1}(V)$. Thus, $f^{-1}\left(M_{2} \ominus V\right) \subseteq f^{-1}\left(M_{2}\right) \ominus f^{-1}(V)$. Also, let $x \in^{k} f^{-1}\left(M_{2}\right) \ominus f^{-1}(V)$. It follows that $f(k / x) \in M_{2}$ and $f(k / x) \notin V$. Consequently, $x \in^{k} f^{-1}\left(M_{2} \ominus V\right)$. Therefore, $f^{-1}\left(M_{2}\right) \ominus f^{-1}(V) \subseteq f^{-1}\left(M_{2} \ominus V\right)$. Hence, $f^{-1}\left(M_{2} \ominus V\right)=f^{-1}\left(M_{2}\right) \ominus f^{-1}(V)$.

(2) Let $x \in^{k} N$. Hence, $f(k / x) \in f(N)$. So $x \in^{k} f^{-1}(f(N))$, and hence, $N \subseteq f^{-1}(f(N))$. Now, let $f$ be one-one and $x \in^{k} f^{-1}(f(N))$. It follows that $f(k / x) \in f(N)$. So there exists $y \in^{r} N$ such that $f(k / x)=f(r / y)$. Since $f$ is one-one, then $k / x=r / y$. Therefore, $x \in^{k} N$. Thus, if $f$ is one-one, then $f^{-1}(f(N)) \subseteq N$.

(3) Let $x \in^{k} f\left(f^{-1}(V)\right)$. It follows that there exists $y \in^{r} f^{-1}(V)$ such that $f(k / x)=f(r / y)$ and $f(r / y) \in V$. So $f(k / x) \in V$. Thus, $f\left(f^{-1}(V)\right) \subseteq V$. Also, if $f$ is onto and $x \in^{k} V$. Hence, $f^{-1}(k / x) \in f^{-1}(V), f$ is onto, so $k / x \in f\left(f^{-1}(V)\right)$. Therefore, if $f$ is onto, then $V \subseteq f\left(f^{-1}(V)\right)$.

Theorem 2 Let $N_{1}$ and $N_{2}$ be submsets of an mset $M$. Then:

(1) If $C_{\left(N_{1} \cap N_{2}\right)}(x)=0$ for all $x \in M^{*}$, then $C_{N_{1}}(x) \leq C_{\left(M \ominus N_{2}\right)}$ (x) for all $x \in M^{*}$.

(2) $C_{N_{1}}(x) \leq C_{N_{2}}(x) \Leftrightarrow C_{\left(M \ominus N_{2}\right)}(x) \leq C_{\left(M \ominus N_{1}\right)}(x)$ for all $x \in M^{*}$.

Proof (1) If $C_{\left(N_{1} \cap N_{2}\right)}(x)=0$ for all $x \in M^{*}$. Since

$C_{\left(N_{1} \cap N_{2}\right)}(x)=\min \left\{C_{N_{1}}(x), C_{N_{2}}(x)\right\}$, then $C_{N_{1}}(x)=0$ or $C_{N_{2}}(x)=0$ for all $x \in M^{*}$. It follows that $C_{N_{1}}(x)+C_{N_{2}}(x) \leq C_{M}(x)$ for all $x \in M^{*}$, and hence, $C_{N_{1}}(x) \leq C_{M}(x)-C_{N_{2}}(x)=C_{\left(M \ominus N_{2}\right)}(x)$ for all $x \in M^{*}$, then the result.

$C_{N_{1}}(x) \leq C_{N_{2}}(x) \Leftrightarrow-C_{N_{2}}(x) \leq-C_{N_{1}}(x) \Leftrightarrow C_{M}(x)-C_{N_{2}}(x) \leq$ $C_{M}(x)-C_{N_{1}}(x) \Leftrightarrow C_{\left(M \ominus N_{2}\right)}(x) \leq C_{\left(M \ominus N_{1}\right)}(x)$ for all $x \in M^{*}$.

The following example shows that the converse of Theorem 2 is not true in general.

Example 1 Let $M=\{2 / a, 4 / b, 5 / c\}, N_{1}=\{1 / a, 1 / b, 2 / c\}$, and $N_{2}=\{1 / a, 1 / b\}$. Hence, $M \ominus N_{2}=\{1 / a, 3 / b, 5 / c\}$. It is clear that $N_{1} \subseteq M \ominus N_{2}$ but $N_{1} \cap N_{2}=\{1 / a, 1 / b\}$.

The following example shows that $N_{1} \ominus N_{2} \neq N_{1} \cap\left(M \ominus N_{2}\right)$ in general.

Example 2 Let $M=\{3 / x, 4 / y\}, N_{1}=\{2 / x, 3 / y\}$, and $N_{2}=\{1 / x, 2 / y\}$. Hence, $M \ominus N_{2}=$ $\{2 / x, 2 / y\}, N_{1} \ominus N_{2}=\{1 / x, 1 / y\}$, and $N_{1} \cap\left(M \ominus N_{2}\right)=\{2 / x, 2 / y\}$.

Definition 20 Let $X$ be an infinite set. Then, $M=\left\{k_{\alpha} / x_{\alpha}: \alpha \in \Lambda\right\}$ be an infinite mset drawn from $X$. That is, the infinite mset $M$ drawn from $X$ is denoted by $M=$ $\left\{k_{1} / x_{1}, k_{2} / x_{2}, k_{3} / x_{3}, \ldots\right\}$. 
Notation 1 The mset space $[X]_{\infty}^{m}$ is the set of all infinite msets whose elements are in $X$ such that no element in the mset occurs more than $m$ times.

It may be noted that the following examples of mset topologies are not tackled before.

Example 3 Let $M \in[X]_{\infty}^{m}$ and $\left\{k_{0} / x_{0}\right\}$ be a simple submset of $M$. Then, the collection $\tau_{\left(k_{0} / x_{0}\right)}=\left\{V \subseteq M: C_{V}\left(x_{0}\right) \geq k_{0}\right\} \cup\{\emptyset\}$ is an M-topology on $M$ called the particular point M-topology.

Example 4 Let $M \in[X]_{\infty}^{m}$ and $\left\{k_{0} / x_{0}\right\}$ be a simple submset of $M$. Then, the collection $\tau_{k_{0} / x_{0}}=\left\{V \subseteq M: C_{V}\left(x_{0}\right)<k_{0}\right\} \cup\{M\}$ is an M-topology on $M$ called the excluded point M-topology.

Example 5 Let $M \in[X]_{\infty}^{m}$. Then, the collection $\tau=\{V \subseteq M: M \ominus V$ is finite $\} \cup\{\emptyset\}$ is an $M$-topology on $M$ called the cofinite $M$-topology.

Example 6 Let $M \in[X]_{\infty}^{m}$ and $N$ be a submset of $M$. Then, the collection $\tau_{(N)}=\{V \subseteq$ $M: C_{N}(x) \leq C_{V}(x)$ for all $\left.x \in M^{*}\right\} \cup\{\emptyset\}$ is an M-topology on $M$.

Example 7 Let $M \in[X]_{\infty}^{m}$ and $N$ be a submset of $M$. Then, the collection $\tau_{N}=\{V \subseteq M$ : $C_{N}(x) \geq C_{V}(x)$ for all $\left.x \in M^{*}\right\} \cup\{M\}$ is an M-topology on $M$.

\section{Filters in multiset context}

Definition 21 An mset filter $\mathcal{F}$ on an mset $M$ is a nonempty collection of nonempty submsets of $M$ with the properties:

$\left(\mathcal{M F} \mathcal{F}_{1}\right) \phi \notin \mathcal{F}$,

$\left(\mathcal{M F}_{2}\right)$ If $N_{1}, N_{2} \in \mathcal{F}$, then $N_{1} \cap N_{2} \in \mathcal{F}$,

$\left(\mathcal{M F}_{3}\right)$ If $N_{1} \in \mathcal{F}$ and $C_{N_{1}}(x) \leq C_{N_{2}}(x)$ for all $x \in M^{*}$, then $N_{2} \in \mathcal{F}$.

It should be noted that $\mathcal{F}$ is an ordinary set whose elements are msets and the multiset filter is abbreviated as an $M$-filter.

Proposition 1 Let $\mathcal{F}$ be an $M$-filter on a nonempty mset $M$. Then:

(1) $M \in \mathcal{F}$,

(2) Finite intersections of members of $\mathcal{F}$ are in $\mathcal{F}$.

Proof The result follows immediately from Definition 21.

Remark 1 It should be noted that the collection of complements of msets in a proper Mfilter is a nonempty collection closed under the operations of subsets and finite unions. Such a collection is called M-ideal [17].

Example $8 P F(M)$ is an $M$-filter on $M$.

Example $9 P^{*}(M)$ is not an $M$-filter. For one thing, the empty set belongs to it. Secondly, it contains the disjoint msets. 
Example 10 Let $\mathcal{F}=\{M\}$. Then, $\mathcal{F}$ is an $M$-filter. This is the smallest $M$-filter one can define on $M$ and is called the indiscrete $M$-filter on $M$.

Example 11 Let $x \in^{k} M$ and $<k / x>=\{N \subseteq M: k / x \in N\}$. Then, $<k / x>$ is an $M$-filter called the principle $M$-filter at $<k / x>$.

Example 12 More generally, let $N$ be a nonempty submset of $M$ and $<N>=\{G \subseteq M$ : $N \subseteq G\}$. Then, $<N>$ is an $M$-filter called the principle $M$-filter at $N$. In addition to that, the indiscrete M-filter is the principle M-filter at $M$.

Example 13 Let $M$ be an infinite mset and $\mathcal{F}=\left\{N \subseteq M: N^{c}\right.$ is a finite $\}$. Then, $\mathcal{F}$ is called the cofinite $M$-filter on $M$.

Example 14 Let $(M, \tau)$ be an M-topological space and $x \in^{k} M$. Then, $\mathcal{N}_{k / x}$ is an M-filter on $M$.

It should be noted that the $M$-filter may contain the submset and it is complement because the intersection between submset and its complement is not necessary empty.

Example 15 Let $M=\{2 / a, 3 / b\}$ and $\mathcal{F}=\{M,\{1 / a\},\{2 / a\},\{1 / a, 1 / b\},\{1 / a, 2 / b\}$, $\{1 / a, 3 / b\},\{2 / a, 1 / b\},\{2 / a, 2 / b\}\}$. It is clear that $\mathcal{F}$ is $a n M$-filter and $\{1 / a\}$ and its complement $\{1 / a, 3 / b\}$ belong to $\mathcal{F}$.

Definition 22 Let $M$ be a nonempty mset and $\mathcal{F}_{1}, \mathcal{F}_{2}$ be two $M$-filters on $M$. Then, $\mathcal{F}_{1}$ is said to be coarser or smaller than $\mathcal{F}_{2}$, denoted by $\mathcal{F}_{1} \leq \mathcal{F}_{2}$, if $\mathcal{F}_{1} \subseteq \mathcal{F}_{2}$, or alternatively $\mathcal{F}_{2}$ is said to be finer or stronger than $\mathcal{F}_{1}$.

Theorem 3 Let $M$ be an mset and $\left\{\mathcal{F}_{i}\right\}, i \in I$ be a nonempty family of $M$-filters on $M$. Then, $\mathcal{F}=\cap_{i \in I} \mathcal{F}_{i}$ is an $M$-filter on $M$.

Proof Since $M \in \mathcal{F}_{i}$ for each $i \in I$, hence $M \in \cap_{i \in I} \mathcal{F}_{i}$; that is, $M \in \mathcal{F}$. Moreover, $\left(\mathcal{M F}_{1}\right)$ implies $\phi \notin \mathcal{F}_{i}$ for each $i \in I$. Therefore, $\mathcal{F}$ be a nonempty collection of a nonempty submsets of $M$. Let $N_{1}, N_{2} \in \mathcal{F}$, then $N_{1}, N_{2} \in \mathcal{F}_{i}$ for each $i \in I$. Since $\mathcal{F}_{i}$ is an $M$-filter for each $i \in I$, hence $\left(\mathcal{M F}_{2}\right)$ implies $N_{1} \cap N_{2} \in \mathcal{F}_{i}$ for each $i \in I$. Thus, $N_{1} \cap N_{2} \in \mathcal{F}$. Now let $N_{1} \in \mathcal{F}$ and $C_{N_{1}}(x) \leq C_{N_{2}}(x)$ for all $x \in M^{*}$. It follows that $N_{1} \in \mathcal{F}_{i}$ for each $i \in I$. Hence , $\left(\mathcal{M F}_{2}\right)$ implies that $N_{2} \in \mathcal{F}_{i}$ for each $i \in I$. Therefore, $N_{2} \in \mathcal{F}$, and hence, the result follows.

The following example shows that the union of two $M$-filters on a nonempty mset $M$ is not necessarily an $M$-filter on $M$.

Example 16 Let $M=\{3 / a, 4 / b, 2 / c, 5 / d\}, \quad \mathcal{F}_{1}=\{M,\{3 / a, 4 / b, 2 / c\}\}$, and $\mathcal{F}_{2}=\{M,\{3 / a, 4 / b, 5 / d\}\}$. Then, $\mathcal{F}_{1} \cup \mathcal{F}_{2}=\{M,\{3 / a, 4 / b, 2 / c\},\{3 / a, 4 / b, 5 / d\}\}$. Although $\mathcal{F}_{1}$ and $\mathcal{F}_{2}$ are two $M$-filters on $M, \mathcal{F}_{1} \cup \mathcal{F}_{2}$ is not $M$-filter. Since $\{3 / a, 4 / b, 2 / c\},\{3 / a, 4 / b, 5 / d\} \in \mathcal{F}_{1} \cup \mathcal{F}_{2}$, but $\{3 / a, 4 / b, 2 / c\} \cap\{3 / a, 4 / b, 5 / d\}=$ $\{3 / a, 4 / b\} \notin \mathcal{F}_{1} \cup \mathcal{F}_{2}$. 


\section{Basis and subbasis in multiset filters}

Definition 23 Let $\mathcal{B}$ be a nonempty collection of a nonempty submsets of $M$. Then, $\mathcal{B}$ is called an $M$-filter basis on $M$ if

$\left(\mathcal{M B}_{1}\right) \phi \notin \mathcal{B}$,

$\left(\mathcal{M B}_{2}\right)$ If $B_{1}, B_{2} \in \mathcal{B}$, then there exists a $B \in \mathcal{B}$ such that $C_{B}(x) \leq C_{\left(B_{1} \cap B_{2}\right)}(x)$ for all $x \in M^{*}$.

Theorem 4 Let $\mathcal{B}$ be an $M$-filter basis on $M$, and $\mathcal{F}$ consists of all msets which are super msets in $\mathcal{B}$; that is, $\mathcal{F}=\left\{N \subseteq M: \forall x \in M^{*} C_{N}(x) \geq C_{N_{1}}(x)\right.$, for some $\left.N_{1} \in \mathcal{B}\right\}$.Then, $\mathcal{F}$ is an $M$-filter on $M$. Furthermore, it is the smallest $M$-filter which contains $\mathcal{B}$. It is called the $M$-filter generated by $\mathcal{B}$.

Proof Since $\mathcal{F}$ consists of all msets which are super msets in $\mathcal{B}$, hence every member of $\mathcal{B}$ is also a member of $\mathcal{F}$. Consequently, $\mathcal{B} \subseteq \mathcal{F}$ and hence $\mathcal{F} \neq \phi$. Since $\mathcal{F}$ contains all submsets of $M$ which contain a member of $\mathcal{B}$ and $\phi \notin \mathcal{B}$, hence $\phi \notin \mathcal{F}$. Thus, $\mathcal{F}$ satisfies $\left(\mathcal{M F}_{1}\right)$. To prove that $\mathcal{F}$ satisfies $\left(\mathcal{M F}_{2}\right)$, let $N_{1}, N_{2} \in \mathcal{F}$. Hence, for all $x \in M^{*}, C_{N_{1}}(x) \geq$ $B_{1}(x)$ and $C_{N_{2}}(x) \geq C_{B_{2}}(x)$ for some $B_{1}, B_{2} \in \mathcal{B}$. It follows that there exists $B \in \mathcal{B}$ such that $C_{B}(x) \leq C_{\left(B_{1} \cap B_{2}\right)}(x)$ for all $x \in M^{*}$ and hence $C_{\left(N_{1} \cap N_{2}\right)}(x) \geq C_{\left(B_{1} \cap B_{2}\right)}(x) \geq C_{B}(x)$ for all $x \in M^{*}$. Consequently, $N_{1} \cap N_{2} \in \mathcal{F}$. For $\left(\mathcal{M F}_{3}\right)$, let $N_{1} \in \mathcal{F}$ and $C_{N_{1}}(x) \leq C_{N_{2}}(x)$ for all $x \in M^{*}$. It follows that for all $x \in M^{*} C_{N_{1}}(x) \geq C_{B}(x)$ for some $B \in \mathcal{B}$. Therefore, for all $x \in M^{*} C_{N_{2}}(x) \geq C_{N_{1}}(x) \geq C_{B}(x)$ for some $B \in \mathcal{B}$. Thus, $N_{2} \in \mathcal{F}$. Hence, $\mathcal{F}$ is an $M$ filter on $M$. Now, let $\mathcal{F}_{1}$ be an $M$-filter which contains $\mathcal{B}$ such that $\mathcal{F}_{1} \leq \mathcal{F}$. Let $N \in \mathcal{F}$. It follows that for all $x \in M^{*} C_{N}(x) \geq C_{N_{1}}(x)$, for some $N_{1} \in \mathcal{B}$. This result, combined with $N_{1} \in \mathcal{F}_{1}$ and $\left(\mathcal{M F}_{3}\right)$, implies $N \in \mathcal{F}_{1}$. Hence, $\mathcal{F} \leq \mathcal{F}_{1}$. Therefore, $\mathcal{F}=\mathcal{F}_{1}$. Thus, $\mathcal{F}$ is the smallest $M$-filter which contains $\mathcal{B}$.

Example 17 Every M-filter is trivially an M-filter basis of itself.

Example $18 \mathcal{B}=\{k / x\}$ is an $M$-filter basis and generates the principle $M$-filter at $k / x$.

Example $19 \mathcal{B}=\{N\}$ is an $M$-filter basis and generates the principle M-filter at $N$.

Example 20 Let $M=\left\{k_{1} / x_{1}, k_{2} / x_{2}, k_{3} / x_{3}, \ldots, k_{m} / x_{n}\right\}$. Then, $\mathcal{B}=\left\{1 / x_{1}, 1 / x_{2}, 1 / x_{3}, \ldots\right.$, $\left.1 / x_{n}\right\}$ is an $M$-filter basis and generates $P F(M)$.

Theorem 5 Let $M$ be a nonempty mset, $\mathcal{B}$ an $M$-filter basis which generates $\mathcal{F}_{1}$, and $\mathcal{B}^{*}$ an $M$-filter basis which generates $\mathcal{F}_{2}$. Then, $\mathcal{F}_{1} \leq \mathcal{F}_{2}$ if and only if every member of $\mathcal{B}$ contains a member of $\mathcal{B}^{*}$.

Proof Suppose $\mathcal{F}_{1} \leq \mathcal{F}_{2}$ and $B \in \mathcal{B}$. Since $\mathcal{B}$ is an $M$-filter basis which generates $\mathcal{F}_{1}$, then $B \in \mathcal{F}_{1}$. Since $\mathcal{F}_{1} \leq \mathcal{F}_{2}$, thus $B \in \mathcal{F}_{2}$, which implies that there exists $B^{*} \in \mathcal{B}^{*}$ such that $C_{B^{*}}(x) \leq C_{B}(x)$ for all $x \in M^{*}$. Therefore, every member of $\mathcal{B}$ contains a member of $\mathcal{B}^{*}$. On the other hand, let every member of $\mathcal{B}$ contain a member of $\mathcal{B}^{*}$ and $F \in \mathcal{F}_{1}$. Since $\mathcal{B}$ is an $M$-filter basis which generates $\mathcal{F}_{1}$, it follows that there exists $B \in \mathcal{B}$ such that $C_{B}(x) \leq C_{F}(x)$ for all $x \in M^{*}$. From the assumption, there exists $B^{*} \in \mathcal{B}^{*}$ such that 
$C_{B^{*}}(x) \leq C_{B}(x) \leq C_{F}(x)$ for all $x \in M^{*}$. This result, combined with $\mathcal{B}^{*}$ is an $M$-filter basis which generates $\mathcal{F}_{2}$, implies $F \in \mathcal{F}_{2}$. Consequently, $\mathcal{F}_{1} \leq \mathcal{F}_{2}$.

Definition 24 The two $M$-filter basis (M-filter subbasis) are $\mathcal{F}$-equivalent if they generate the same $M$-filter.

Theorem 6 Let $\mathcal{B}$ and $\mathcal{B}^{*}$ be an $M$-filter basis on a nonempty mset $M$. Then, $\mathcal{B}$ and $\mathcal{B}^{*}$ are equivalent if and only if every member of $\mathcal{B}$ contains a member of $\mathcal{B}^{*}$ and every member of $\mathcal{B}^{*}$ contains a member of $\mathcal{B}$.

Proof The result follows immediately from Theorem 4 .

Theorem 7 Let $M_{1}$ and $M_{2}$ be two nonempty msets drawn from $X$ and $Y$, respectively, $f: M_{1} \rightarrow M_{2}$ be an mset function, $\mathcal{B}$ be an $M$-filter basis on $M_{1}$, and $\mathcal{B}^{*}$ be an $M$-filter basis on $M_{2}$. Then:

(1) $K_{1}=\{f(B): B \in \mathcal{B}\}$ is an $M$-filter basis on $M_{2}$.

(2) If every member of $\mathcal{B}^{*}$ intersects $f\left(M_{1}\right)$, then $K_{2}=\left\{f^{-1}\left(B^{*}\right): B^{*} \in \mathcal{B}^{*}\right\}$ is an $M$-filter basis on $M_{1}$.

Proof (1) Since $\mathcal{B}$ is an $M$-filter basis on $M_{1}$. It follows that $\mathcal{B} \neq \phi$. So, $K_{1} \neq \phi$. For $\left(\mathcal{M} \mathcal{B}_{1}\right)$, since $\phi \notin \mathcal{B}$, hence $\phi \notin K_{1}$. To prove $\left(\mathcal{M} \mathcal{B}_{2}\right)$, let $f\left(B_{1}\right), f\left(B_{2}\right) \in \mathcal{B}$ such that $B_{1}, B_{2} \in \mathcal{B}$. Since $\mathcal{B}$ is an $M$-filter basis on $M_{1}$, it follows that there exists $B \in \mathcal{B}$ such that $C_{B}(x) \leq C_{\left(B_{1} \cap B_{2}\right)}(x)$ for all $x \in M^{*}$. Thus, $C_{f(B)}(y) \leq C_{f\left(B_{1} \cap B_{2}\right)}(y) \leq C_{f\left(B_{1}\right) \cap f\left(B_{2}\right)}(y)$ for all $y \in Y$. Therefore, there exists $f(B) \in K_{1}$ such that $C_{f(B)}(y) \leq C_{f\left(B_{1}\right) \cap f\left(B_{2}\right)}(y)$ for all $y \in Y$. Hence, $K_{1}$ is an $M$-filter basis on $M_{2}$.

(2) The proof is similar to part (1).

\section{Multiset ultrafilter}

Definition 25 An $M$-filter $\mathcal{F}$ is called an mset ultrafilter on $M$, M-ultrafilter, if there is no strictly finer $M$-filter than $\mathcal{F}$. That is, if $\mathcal{F}^{*}$ is an $M$-ultrafilter and $\mathcal{F}^{*} \geq \mathcal{F}$, then $\mathcal{F}^{*}=\mathcal{F}$.

Example 21 Let $M=\{2 / a, 3 / b\}$. Then, $\mathcal{F}_{1}=\{M,\{1 / a\},\{2 / a\},\{1 / a, 1 / b\}$, $\{1 / a, 2 / b\},\{1 / a, 3 / b\},\{2 / a, 1 / b\},\{2 / a, 2 / b\}\}$ and $\mathcal{F}_{2}=\{M,\{1 / b\},\{2 / b\},\{3 / b\},\{1 / a, 1 / b\}$, $\{2 / a, 1 / b\},\{1 / a, 2 / b\},\{2 / a, 2 / b\},\{1 / a, 3 / b\},\{2 / a, 3 / b\}\}$ are $M$-ultrafilters on $M$.

Theorem 8 Let $M$ be a nonempty mset. An $M$-filter $\mathcal{F}$ is an $M$-ultrafilter if it contains all submsets of $M$ which intersects every member of $\mathcal{F}$.

Proof Let $\mathcal{F}$ be an $M$-ultrafilter on $M$ and $N$ be a submset of $M$ such that $F \cap N \neq \phi$ for all $F \in \mathcal{F}$. Now, we want to show that the collection $\mathcal{F}^{*}=\left\{F^{*}: \forall x \in M^{*} C_{F^{*}}(x) \geq\right.$ $C_{(N \cap F)}(x)$ for some $\left.F \in \mathcal{F}\right\}$ is an $M$-filter on $M$. For $\left(\mathcal{M F}_{1}\right)$, since $C_{(N \cap F)}(x) \geq C_{\phi}(x)$ for all $x \in M^{*}$. Thus, $\phi \notin \mathcal{F}^{*}$. For $\left(\mathcal{M F}_{2}\right)$, let $F_{1}^{*}, F_{2}^{*} \in \mathcal{F}^{*}$. Hence, for all $x \in M^{*}$ $C_{F_{1}^{*}}(x) \geq C_{\left(N \cap F_{1}\right)}(x)$ and $C_{F_{2}^{*}}(x) \geq C_{\left(N \cap F_{2}\right)}(x)$ for some $F_{1}, F_{2} \in \mathcal{F}$. It follows that for 
all $x \in M^{*} C_{\left(F_{1}^{*} \cap F_{2}^{*}\right)}(x) \geq C_{\left[N \cap\left(F_{1} \cap F_{2}\right)\right]}(x)$. Therefore, $F_{1}^{*} \cap F_{2}^{*} \in \mathcal{F}^{*}$. To prove that $\mathcal{F}^{*}$ satisfies $\left(\mathcal{M F}_{3}\right)$, let $F_{1}^{*} \in \mathcal{F}^{*}$ and $C_{F_{1}^{*}}(x) \leq C_{F_{2}^{*}}(x)$ for all $x \in M^{*}$. It follows that for all $x \in M^{*} C_{F_{2}^{*}}(x) \geq C_{F_{1}^{*}}(x) \geq C_{(N \cap F)}(x)$ for some $F \in \mathcal{F}$. Consequently, $F_{2}^{*} \in \mathcal{F}^{*}$. Hence, $\mathcal{F}^{*}$ is an $M$-filter on $M$. Since $C_{F}(x) \geq C_{(N \cap F)}(x)$ for all $x \in M^{*}$, then $\mathcal{F}^{*} \geq \mathcal{F}$. Since $\mathcal{F}$ is an $M$-ultrafilter on $M$, it follows that $\mathcal{F}^{*}=\mathcal{F}$. Moreover, $N \in \mathcal{F}$ as for all $x \in M^{*}$ $C_{N}(x) \geq C_{(N \cap F)}(x)$.

Theorem 9 Let $\mathcal{F}$ be an $M$-ultrafilter on a nonempty mset $M$. Then, for each $N \subseteq M$, either $N$ or $N^{c} \in \mathcal{F}$.

Proof Let $\mathcal{F}$ be an $M$-ultrafilter on $M$ and $N \subseteq M$. If there exists $F \in \mathcal{F}$ such that $C_{(F \cap N)}(x)=0$ for all $x \in M^{*}$, then Theorem 2 part (1) implies $C_{F}(x) \leq C_{N^{c}}(x)$ for all $x \in M^{*}$. Thus, $N^{c} \in \mathcal{F}$. Otherwise, $C_{(F \cap N)}(x)>0$ for all $x \in M^{*}$. Thus, Theorem 8 implies $N \in \mathcal{F}$, then the result.

The following example shows that the converse of Theorem 9 is incorrect in general.

Example 22 Let $M=\{2 / a, 3 / b\}$. Then, $\mathcal{F}=\{M,\{2 / b\},\{3 / b\},\{1 / a, 2 / b\},\{1 / a, 3 / b\}$, $\{2 / a, 2 / b\},\{2 / a, 1 / b\},\{2 / a, 2 / b\}\}$ is an $M$-filter on $M$. Although for each $N \subseteq M$, either $N$ or $N^{c} \in \mathcal{F}, \mathcal{F}$ is not M-ultrafilter. As $\mathcal{F}^{*}=\{M,\{1 / b\},\{2 / b\},\{3 / b\},\{1 / a, 1 / b\}$, $\{1 / a, 2 / b\},\{1 / a, 3 / b\},\{2 / a, 1 / b\},\{2 / a, 2 / b\},\{2 / a, 3 / b\}\}$ is finer than $\mathcal{F}$.

Theorem 10 Let $\mathcal{F}$ be an M-ultrafilter on a nonempty mset $M$. Then, for each two nonempty submsets $N_{1}, N_{2}$ of $M$ such that $N_{1} \cup N_{2} \in \mathcal{F}$, either $N_{1} \in \mathcal{F}$ or $N_{2} \in \mathcal{F}$.

Proof Assume $N_{1} \cup N_{2} \in \mathcal{F}$ and $N_{1} \in \mathcal{F}$ and $N_{2} \in \mathcal{F}$. Define $\mathcal{F}^{*}=\{G \subseteq M$ : $G \cup N_{2} \in \mathcal{F}$ \}. Now, we want to prove that $\mathcal{F}^{*}$ is an $M$-filter on $M$. Since $N_{1} \cup N_{2} \in \mathcal{F}$, then $N_{1} \in \mathcal{F}^{*}$. Hence, $\mathcal{F}^{*} \neq \phi$. For $\left(\mathcal{M F}_{1}\right)$, since $\phi \cup N_{2}=N_{2} \notin \mathcal{F}^{*}$, it follows that $\phi \notin \mathcal{F}^{*}$. To prove that $\mathcal{F}^{*}$ satisfies $\left(\mathcal{M F}_{2}\right)$, let $G_{1}, G_{2} \in \mathcal{F}^{*}$. Hence, $G_{1} \cup N_{2} \in \mathcal{F}$ and $G_{2} \cup N_{2} \in \mathcal{F}$. Thus, $\left(G_{1} \cup N_{2}\right) \cap\left(G_{2} \cup N_{2}\right) \in \mathcal{F}$. Therefore, $\left(G_{1} \cap G_{2}\right) \cup N_{2} \in$ $\mathcal{F}$. Hence, $G_{1} \cap G_{2} \in \mathcal{F}$. For $\left(\mathcal{M F}_{3}\right)$, let $G_{1} \in \mathcal{F}^{*}$ and $C_{G_{1}}(x) \leq C_{G_{2}}(x)$ for all $x \in$ $M^{*}$. Hence, $G_{1} \cup N_{2} \in \mathcal{F}$ and $C_{\left(G_{1} \cup N_{2}\right)}(x) \leq C_{\left(G_{2} \cup N_{2}\right)}(x)$ for all $x \in M^{*}$. Thus, $G_{2} \cup$ $N_{2} \in \mathcal{F}$. Hence, $G_{2} \in \mathcal{F}^{*}$. Consequently, $\mathcal{F}^{*}$ is an $M$-filter on $M$. Let $F \in \mathcal{F}$. Since $C_{F}(x) \leq C_{\left(F \cup N_{2}\right)}(x)$ for all $x \in M^{*}$, then $\left(\mathcal{M F}_{3}\right)$ implies $F \cup N_{2} \in \mathcal{F}$. Therefore, $F \in \mathcal{F}^{*}$; that is, $\mathcal{F} \leq \mathcal{F}^{*}$. But $\mathcal{F}$ is an $M$-ultrafilter. Thus, there is a contradiction. Therefore, $N_{1} \in \mathcal{F}$ or $N_{2} \in \mathcal{F}$.

The following example shows that the converse of Theorem 10 is wrong in general.

Example 23 Let $M=\{3 / a, 4 / b\}$ and $\mathcal{F}=\{M,\{3 / a\},\{3 / a, 1 / b\},\{3 / a, 2 / b\},\{3 / a, 3 / b\}\}$ be an M-filter on $M$. Although for all $N_{1}, N_{2} \subseteq M$ such that $N_{1} \cup N_{2} \in$ $\mathcal{F}$, either $N_{1} \in \mathcal{F}$ or $N_{2} \in \mathcal{F}, \mathcal{F}$ is not M-ultrafilter. As $\mathcal{F}^{*}=$ $\{M,\{3 / a\},\{4 / b\},\{3 / a, 1 / b\},\{3 / a, 2 / b\},\{3 / a, 3 / b\},\{1 / a, 4 / b\},\{2 / a, 4 / b\}\}$ is finer than $\mathcal{F}$.

\section{Convergence of multiset filters}

Definition 26 Let $(M, \tau)$ be an M-topological space and $\mathcal{F}$ be an $M$-filter on $M . \mathcal{F}$ is said to $\tau$-converge to $k / x$ (written $\mathcal{F} \stackrel{\tau}{\longrightarrow} k / x$ ) if $\mathcal{N}_{k / x} \subseteq \mathcal{F}$; that is, if $\mathcal{F} \geq \mathcal{N}_{k / x}$. 
Example 24 For each mpoint, $k / x, \mathcal{N}_{k / x}$ converges to $k / x$.

Example 25 Let $\tau$ be the cofinite $M$-topology on $M$ and $\mathcal{F}$ be the cofinite $M$-filter. Then, $\mathcal{F}$ converges to each mpoint.

Example 26 Let $(M, \tau)$ be the indiscrete $M$ - topology and $\mathcal{F}$ any $M$-filter on $M$; then, $\mathcal{F}$ converges to each mpoint.

Theorem 11 Let $(M, \tau)$ be an M-topological space and $\mathcal{F}$ and $\mathcal{F}^{*}$ be $M$-filters on $M$ such that $\mathcal{F}^{*} \geq \mathcal{F}$. If $\mathcal{F} \stackrel{\tau}{\longrightarrow} k / x$, then $\mathcal{F}^{*} \stackrel{\tau}{\longrightarrow} k / x$.

Proof Since $\mathcal{F} \stackrel{\tau}{\longrightarrow} k / x$ and $\mathcal{F}^{*} \geq \mathcal{F}$, it follows that $\mathcal{F}^{*} \geq \mathcal{F} \geq \mathcal{N}_{k / x}$. Hence, Definition 26 implies that $\mathcal{F}^{*} \stackrel{\tau}{\longrightarrow} k / x$.

Theorem 12 Let $\left(M, \tau_{1}\right)$ and $\left(M, \tau_{2}\right)$ be two M-topological spaces such that $\tau_{2} \leq \tau_{1}$ and $\mathcal{F}$ be an $M$-filter on $M$ such that $\mathcal{F} \stackrel{\tau_{1}}{\longrightarrow} k / x$. Then, $\mathcal{F} \stackrel{\tau_{2}}{\longrightarrow} k / x$.

Proof Since $\tau_{2} \leq \tau_{1}$ and $\mathcal{F} \stackrel{\tau_{1}}{\longrightarrow} k / x$, it follows that $\mathcal{N}_{k / x}^{\tau_{2}} \leq \mathcal{N}_{k / x}^{\tau_{1}}$ and $\mathcal{N}_{k / x}^{\tau_{1}} \leq \mathcal{F}$. Thus, $\mathcal{N}_{k / x}^{\tau_{2}} \leq \mathcal{N}_{k / x}^{\tau_{1}} \leq \mathcal{F}$. Hence, Definition 26 implies $\mathcal{F} \stackrel{\tau_{2}}{\longrightarrow} k / x$, then the result.

Theorem 13 Let $(M, \tau)$ be an M-topological space, then the following assertions are equivalent:

(1) $\mathcal{F} \stackrel{\tau}{\longrightarrow} k / x$,

(2) Every $M$-ultrafilter containing $\mathcal{F}$ converges to $k / x$.

Proof On the one hand, let $\mathcal{F}^{*}$ be an $M$-ultrafilter containing $\mathcal{F}$; that is, $\mathcal{F} \leq \mathcal{F}^{*}$. This result, combined with assertion (1), implies $\mathcal{N}_{k / x} \leq \mathcal{F} \leq \mathcal{F}^{*}$. Thus, Definition 26 implies $\mathcal{F}^{*} \stackrel{\tau}{\longrightarrow} k / x$. Hence, (1) implies (2). On the other hand, (2) implies that $\mathcal{N}_{k / x}$ is contained in every $M$-ultrafilter containing $\mathcal{F}$. Hence, $\mathcal{N}_{k / x}$ is contained in the intersection of all $M$-ultrafilter containing $\mathcal{F}$. This result, combined with $\mathcal{F}$ is the intersection of all $M$ ultrafilter containing $\mathcal{F}$, implies $\mathcal{N}_{k / x} \leq \mathcal{F}$. Then, $\mathcal{F} \stackrel{\tau}{\longrightarrow} k / x$. Hence, (2) implies (1).

Theorem 14 Let $(M, \tau)$ be an $M$-topological space and $N$ be a nonempty submset of $M$; then, the following assertions are equivalent:

(1) $\quad N \in \tau$,

(2) If $\mathcal{F} \stackrel{\tau}{\longrightarrow} k / x$ such that $x \in^{k} N$, then $N \in \mathcal{F}$.

Proof The first direction is a direct consequence of Definition 26 and assertion (1). On the other hand, let $x \in^{m} N$. Then Example 24 shows that $\mathcal{N}_{m / x} \stackrel{\tau}{\longrightarrow} m / x$. Thus, assertion (2) implies that $N \in \mathcal{N}_{m / x}$; that is, $N$ is a neighborhood of $m / x$. Hence, $N$ is a neighborhood for every $x \in{ }^{k} N$. Thus, $N \in \tau$; that is, (2) implies (1).

\section{Abbreviations}

mset: Multiset; M-filter: multiset filter; : M-ultrafilter: multiset ultrafilter

Acknowledgements

Not applicable.

Authors' contributions

All authors jointly worked on the results, and they read and approved the final manuscript 
Funding

Not applicable

Availability of data and materials

Not applicable

\section{Competing interests}

The authors declare that they have no competing interests.

\section{Author details}

${ }^{1}$ Doctoral School of Mathematical and Computational Sciences, University of Debrecen, Pf. 400, Debrecen, H-4002, Hungary. ${ }^{2}$ Department of Mathematics, Faculty of Education, Ain Shams University, Cairo 11341, Egypt. ${ }^{3}$ Department of Mathematics, National Institute of Technology Calicut, Calicut 673601 , Kerala, India. ${ }^{4}$ Department of Mathematics, MES Kalladi College Mannarkkad, Palakkad 678 583, Kerala, India.

Received: 23 July 2019 Accepted: 21 November 2019

Published online: 16 December 2019

\section{References}

1. Blizard, W. D.: Multiset theory. Notre Dame J. Form. Log. 30, 36-65 (1989)

2. Yager, R. R.: On the theory of bags. Int. J. Gen. Syst. 13, 23-37 (1986). https://doi.org/10.1080/03081078608934952

3. Blizard, W. D.: Negative Membership. Notre Dame J. Form. Log. 31, 346-368 (1990)

4. Jena, S. P., Ghosh, S. K., Tripathy, B. K.: On the theory of bags and lists. Inform. Sci. 132, 241-254 (2001). https://doi. org/10.1016/S0020-0255(01)00066-4

5. Girish, K. P., John, S. J.: General relations between partially ordered multisets and their chains and antichains. Math. Commun. 14, 193-206 (2009)

6. Girish, K. P., John, S. J.: Relations and functions in multiset context. Inform. Sci. 179, 758-768 (2009). https://doi.org/ 10.1016/j.ins.2008.11.002

7. Girish, K. P., John, S. J.: Multiset topologies induced by multiset relations. Inform. Sci. 188, 298-313 (2012). https://doi. org/10.1016/j.ins.2011.11.023

8. Girish, K. P., John, S. J.: On multiset topologies. Theory Appl. Math. Comput. Sci. 2, 37-52 (2012)

9. Kandil, A, Tantawy, O. A, El-Sheikh S.A., Zakaria, A.: Multiset proximity spaces. J. Egypt. Math. Soc. 24, $562-567$ (2016). https://doi.org/10.1016/j.joems.2015.12.002

10. Osmanoğlu, I., Tokat, D.: Connectedness on soft multi topological spaces. J. New Results Sci. 2, 8-18 (2013)

11. Osmanoğlu, I., Tokat, D.: Compact soft multi spaces. Eur. J. Pure Appl. Math. 7, 97-108 (2014)

12. Syropoulos, A.: Mathematics of multisets. Multiset Process. LNCS. 2235, 347-358 (2001). https://doi.org/10.1007/3540-45523-X_17

13. Syropoulos, A.: Categorical models of multisets. Rom. J. Inf. Sci. Technol. 6, 393-400 (2003)

14. Singh, D., Ibrahim, A. M., Yohanna, T., Singh, J. N.: An overview of the applications of multisets. Novi Sad J. Math. $\mathbf{3 7}$ 73-92 (2007)

15. Singh, D., Singh, J. N.: A note on the definition of multisubset, Association for Symbolic Logic. USA, Annual Conference (2007)

16. El-Sheikh, S. A., Zakaria, A.: Note on rough multiset and its multiset topology. Ann. Fuzzy Math. Inf. 10, 235-238 (2015)

17. Zakaria, A., John, S. J., El-Sheikh, S. A.: Generalized rough multiset via multiset ideals. Intell. Fuzzy Syst. 30, 1791-1802 (2016). https://doi.org/10.3233/IFS-151891

\section{Publisher's Note}

Springer Nature remains neutral with regard to jurisdictional claims in published maps and institutional affiliations.

\section{Submit your manuscript to a SpringerOpen ${ }^{\circ}$ journal and benefit from:}

- Convenient online submission

Rigorous peer review

- Open access: articles freely available online

- High visibility within the field

- Retaining the copyright to your article

Submit your next manuscript at $\gg$ springeropen.com 